This item was submitted to Loughborough's Research Repository by the author.

Items in Figshare are protected by copyright, with all rights reserved, unless otherwise indicated.

\title{
Using data envelopment analysis for the assessment of technical efficiency of units with different specialisations: an application to agriculture
}

\section{PLEASE CITE THE PUBLISHED VERSION}

http://dx.doi.org/10.1016/j.omega.2015.01.015

\section{PUBLISHER}

(C) Elsevier B.V.

\section{VERSION}

AM (Accepted Manuscript)

\section{PUBLISHER STATEMENT}

This work is made available according to the conditions of the Creative Commons Attribution-NonCommercialNoDerivatives 4.0 International (CC BY-NC-ND 4.0) licence. Full details of this licence are available at: https://creativecommons.org/licenses/by-nc-nd/4.0/

\section{LICENCE}

CC BY-NC-ND 4.0

\section{REPOSITORY RECORD}

Atici, Kazin Baris, and Victor V. Podinovski. 2019. "Using Data Envelopment Analysis for the Assessment of Technical Efficiency of Units with Different Specialisations: An Application to Agriculture”. figshare. https://hdl.handle.net/2134/18170. 


\title{
Using data envelopment analysis for the assessment of technical efficiency of units with different specialisations: an application to agriculture
}

\author{
Kazim Baris Atici \\ Department of Business Administration, Hacettepe University, Ankara, Turkey \\ kba@hacettepe.edu.tr
}

Phone: +90 3122978700 - 121; Fax: +90 3122992055

\section{Victor V. Podinovski}

Warwick Business School, University of Warwick, Coventry CV4 7AL, UK

v.podinovski@warwick.ac.uk

Phone: +44 (0) 247652 8220; Fax: +44 (0) 2476524539

\begin{abstract}
In this paper we consider the use of data envelopment analysis (DEA) for the assessment of efficiency of units whose output profiles exhibit specialisation. An example of this is found in agriculture where a large number of different crops may be produced in a particular region, but only a few farms actually produce each particular crop. Because of the large number of outputs, the use of conventional DEA models in such applications results in a poor efficiency discrimination. We overcome this problem by specifying production trade-offs between different outputs, relying on the methodology of Podinovski (2004). The main idea of our approach is to relate various outputs to the production of the main output. We illustrate this methodology by an application of DEA involving agricultural farms in different regions of Turkey. An integral part of this application is the elicitation of expert judgements in order to formulate the required production trade-offs. Their use in DEA models results in a significant improvement of the efficiency discrimination. The proposed methodology should also be of interest to other applications of DEA where units may exhibit specialization, such as applications involving hospitals or bank branches.
\end{abstract}

Keywords: data envelopment analysis; trade-offs; weight restrictions; non-homogeneity; agriculture 


\section{Introduction}

One of common challenges in applications of data envelopment analysis (DEA) is the low discriminating power of the models used - this is the ability of the DEA models to differentiate between good and bad performing decision making units (DMUs) by reflecting their performance in a sufficiently wide range of efficiency scores (Cooper et al. 2007; Thanassoulis et al., 2008). It is well-known that the discrimination of a DEA model depends on a number of factors, including the number of inputs and outputs in relation to the number of units, the type (variable or constant) of returns-to-scale assumed (VRS and CRS, respectively) and, more generally, the particular data set that is under the investigation (Angulo-Meza and Lins, 2002; Podinovski and Thanassoulis, 2007).

In this paper we consider another contributing factor that negatively affects the discriminating power of DEA models - the specialisation of DMUs in the production of different subsets of the full set of outputs. According to a recent study by Liu et al. (2013), the three largest areas of reported applications of DEA are banking, health care and agriculture. Examples of specialism can easily be found in all of these fields.

Indeed, in healthcare applications of DEA, hospitals often have different specialisations (in terms of treatments available), and may also have other non-clinical outputs reflecting their engagement in research, education and community services (Olesen and Petersen, 2002; Ozcan, 2008). In banking applications, bank branches may offer a full or reduced range of services - e.g. bank branches on university campuses or at airports may not offer mortgages or home-improvement loans (Golany and Storbeck, 1999; Thanassoulis, 1999).

In agriculture the problem of specialisation of units (agricultural farms) is ubiquitous, and is further exacerbated by a large number of possible farm outputs. Indeed, it is common for farms from the same geographical region to produce a variety of different crops and livestock products. While the majority of farms (but not necessarily all of them) may produce several common outputs, e.g. wheat or potatoes, there are usually many other crops each produced only by a small number of farms.

Theoretically, it is clear why DMUs with specialised profiles are often shown as efficient, or almost efficient, by standard DEA models: such DMUs have very few, if any, comparators among the other DMUs. In particular, if a DMU produces an output that no other unit does, then such a DMU cannot be outperformed by any combination of the other 
units, and by definition is considered efficient regardless of the levels of its other outputs and inputs.

\subsection{Existing approaches}

The DEA literature suggests several ways of dealing with applications in which DMUs have different specialisations, or production profiles.

Removal of outliers and clustering. If the number of specialised DMUs is small, these can be removed from the data set as outliers, together with their specialised outputs. For example, Golany and Storbeck (1999) remove the banks that perform unique activities from their data set. It may also be possible to cluster units by their specialism (as well as other characteristics such as scale and location), as discussed in Thanassoulis (1999). Both techniques may be acceptable on the grounds of homogeneity, i.e. making an assumption that the units in different clusters employ a different technology or operate in different environments. This does not, however, increase (and in fact reduces) the number of comparators for the remaining units, and therefore does not improve the discrimination of the model. The two described approaches are of little use in agricultural applications of DEA where the number of clusters of farms that produce the same sets of outputs may be too high, with few farms in each cluster.

Using only the most common outputs. In some agricultural applications of productivity analysis, including DEA, only the most common and important farm outputs are included in the model, such as rice in Ray and Bhadra (1993), pork products (Galanopoulos et al. 2006), and cereals and oilseed in Luik et al. (2009). A potential problem with this approach is that, if farms produce other outputs in any significant quantities, they will not be able to justify their resources within the technology in which such outputs are omitted, and this approach would favour the farms that produce only the outputs specified in the model. In principle, this problem may be overcome by using as the inputs only the proportion of all resources that have been used in the production of the selected farm outputs. In reality, this may be complicated by the fact that the resources are often reported in an aggregate form, and any disaggregation requires additional assumptions (Iráizoz et al., 2003).

Aggregation using output prices. Agricultural applications of DEA and other efficiency and productivity assessment methods often aggregate different farm outputs using their unit prices, into a value (revenue) output (Färe et al. 1985; Oude Lansink et al., 2002; 
Paul et al., 2004; Davidova and Latruffe, 2007; Speelman et al. 2008; Skevas et al., 2012). While addressing the criticisms with the above two approaches (clustering and the use of only the main common outputs), the aggregation using prices is not without problems itself.

- The aggregation of individual outputs (or inputs) into a single value dimension using price information changes the nature of assessment from the measurement of technical efficiency of units to the measurement of their allocative efficiency (Thomas and Tauer, 1994; Galanopoulos et al. 2006). The latter is theoretically generally lower than the former (Cooper et al., 2007). If the technical efficiency is the main objective of the comparative study, the assessment of allocative efficiency instead of it will generally create an underestimation bias (Thomas and Tauer, 1994).

- The aggregation of outputs into a single dimension forgoes much of the information that might otherwise be observed from the original full-dimensional efficient frontier. For example, it becomes impossible to assess the range of shadow prices of individual outputs and based on them rates and elasticities of substitution and transformation between different outputs and/or inputs (Podinovski and Førsund, 2010; Atici and Podinovski, 2012).

- It is not necessarily clear what prices should be used for the aggregation of different outputs. In agriculture, these are often the prices that the farmers in the region are paid for their products. As noted by Acquaye et al. (2002), such prices may be policy-distorted and the whole aggregation approach is "somewhat questionable, but standard". An alternative is to use the market prices where available. However, in the global economy the prices may fluctuate and be influenced by the droughts, increased demand and other events affecting the major world producers and markets. This may considerably affect the monetary value of agricultural production of individual farms and misrepresent their actual technical performance.

- This aggregation method cannot be used in most public sector applications such as health care, where unit prices of outputs generally do not exist.

Using weight restrictions. Weight restrictions are additional constraints on the input and output weights incorporated in multiplier DEA models. These are often used as a means to improve the discrimination of DEA models (Allen et al., 1997; Thanassoulis, 2001). In particular, weight restrictions may be used in the analysis of DMUs with specialised production profiles - an example is an application to Danish hospitals reported by Olesen and Petersen (2002). Because weight restrictions are usually constructed (or assessed) based on 
value judgements, their use generally results in an unsubstantiated enlargement of the production technology and leads to a well-known drawback: namely, this generally invalidates the meaning of efficiency as a technologically feasible improvement factor (Allen et al., 1997).

\subsection{Proposed methodology}

In this paper we suggest that the problem arising from the specialisation of DMUs with a large overall number of outputs can successfully be overcome by the use of the tradeoff approach to DEA (Podinovski, 2004). We illustrate our development by an application involving wheat-producing farms in Turkey.

The main idea is to relate the production of different outputs to the main output by specifying the production (technological) trade-offs between them, in the form of lower and upper bounds. In our application we relate all specialist crops to the production of the main crop - wheat. The trade-offs are estimates of the use of resources required for the production of specialist crops in relation to the production of wheat. An example of a trade-off taken from our application is as follows: the resources required for the production of 1 tonne of wheat are definitely sufficient for the production of at least 0.75 tonnes of barley, at any farm in the region.

The application to Turkish agriculture showed that the use of the suggested methodology resulted in good efficiency discrimination among the farms. In some regions of Turkey the models with trade-offs discriminated well even if the number of inputs and outputs was larger than the number of farms. In contrast, in these regions all or almost all farms were efficient in the conventional VRS and CRS models.

In comparison to the existing methods discussed above, the proposed methodology has several advantages.

- There is no need for the clustering of units, removal of outliers or specialist outputs for the sole purpose of reducing the dimensionality and improving the efficiency discrimination. (There may nevertheless be other reasons for doing so, for example, concerning the homogeneity of environment or unreliable and missing data.)

- In comparison to the aggregation based on prices, the resulting technology preserves all of the original (disaggregated) output dimensions. The DEA models based on it measure the technical efficiency of DMUs, and not the allocative efficiency (although the objective functions of such models can obviously be adapted for the allocative efficiency 
too). The technology obtained with production trade-offs allows for the analysis of shadow prices of individual inputs and outputs and other measures based on it.

- The proposed methodology is mathematically equivalent to the use of weight restrictions, but there is an important advantage to the former. Although production trade-offs are the dual forms of weight restrictions (Podinovski, 2004), the elicitation of production tradeoffs is performed in the language of the envelopment model. Unlike weight restrictions, the use of production trade-offs results in a meaningful expansion of the technology: all new points added to the technology are explicitly (by the nature of expert judgements) assumed to be producible. This overcomes the noted drawback of the traditional weight restrictions. Namely, in the models with production trade-offs, the efficient targets of inefficient DMUs are producible, and the efficiency measure retains the meaning of the ultimate technologically feasible improvement factor.

It is worth emphasising that the proposed use of production trade-offs (as additional assumptions or information about the production process) leads to a better-informed model of technology than the standard VRS or, respectively, CRS models. The improved discrimination on efficiency is a consequence of using a production model enlarged in a meaningful and controlled way.

The above observation distinguishes the proposed approach from several established methodologies aimed primarily at the improved discrimination of DEA models, without changing the underlying model of technology. One of such methodologies is based on the notion of cross-efficiency - the efficiency indicator calculated for each unit using the optimal weights of other units (Doyle and Green, 1994; Ramón et al., 2011). Another utilises the concept of super-efficiency - a measure of influence of an efficient DMU on the shape of the efficient frontier in its neighbourhood (Andersen and Petersen, 1993). Neither of these approaches involves the construction of a new model of technology. Instead, both methods rely on additional metrics obtained from the standard VRS and CRS DEA models ${ }^{1}$.

Also worth mentioning are multivariate methods and related statistical approaches, such as the combination of principal component analysis (PCA) and DEA, or the omission of variables based on correlation analysis - see a review in Adler and Yazhemsky (2010). This group of methods aims at the reduction of the number of inputs and outputs in a statistically acceptable way. Such methods are based on additional, explicit or implicit, assumptions

\footnotetext{
${ }^{1}$ In principle, the ideas of cross-efficiency and super-efficiency could be used with our extended model of technology, although we do not pursue this further in our paper.
} 
about the data generation process or about the acceptability of removing, or modifying, the inputs and outputs that satisfy certain statistical tests. This is different to the proposed approach which keeps the inputs and outputs intact and does not rely on statistical assumptions.

\subsection{Contribution}

The general notion of production trade-offs and DEA models based on them were defined in Podinovski (2004). However, the practical meaning of trade-offs, the feasibility of their elicitation in different application areas and any tangible difference they could bring to the analysis of efficiency have largely remained to be explored. Our paper addresses this gap in the context of agricultural farms and gives the above theoretical development a practical meaning.

Several specific contributions of our development are worth emphasising.

First, we suggest that the problem of specialisation of DMUs can be overcome by the use of production trade-offs in DEA models, even if the total number of outputs is very large. As outlined, this approach has important advantages over the existing methods and does away with most of their criticisms.

Second, we show that the trade-off methodology can be successfully applied in the context of agricultural farms and illustrate this by an application to wheat-producing farms in Turkey. The approach developed in our paper is based on the comparison of different crops to the main crop in the selected regions (wheat). We also discuss the questions that we asked the experts, and show how the obtained expert judgements are translated to the required format of trade-offs. The resulting DEA models exhibit a good discrimination on efficiency with a full range of various outputs (crops) and high level of specialisation of farms.

Third, within the reported application, we obtain production trade-offs between wheat and 33 other crops produced by farms in different regions of Turkey. If the proposed methodology is used in other similar regions (e.g. in Southern Europe), these trade-offs would need to be confirmed but should be usable at least for initial reference ${ }^{2}$.

\footnotetext{
${ }^{2}$ Note that each trade-off represents a relative measure that compares the amount of wheat and a specialist crop that can be produced given the same amount of resources. If in another region the geographical environment is similar, but the labour, capital and production costs are different, this is likely to affect the inputs of both crops in the same way and keep them equal (although at a different level). Therefore the production trade-off between the two crops is likely to remain relatively unaffected. However, the trade-off should change if, for example, the yields of wheat and the specialist crop are in different proportions in the two regions because of the different environments.
} 
Fourth, the biggest challenge for the proposed methodology is its reliance on confident expert estimates of production trade-offs. Our study shows that such estimates can be obtained by posing simplified questions to experts, without a need of explaining the mathematics behind the DEA models. To put this issue in perspective, our experience shows that this is not more difficult than the assessment of traditional weight restrictions based on value judgements.

Finally, the proposed methodology should not be specific to agriculture and could be used in applications of DEA in other areas, for example in health services. Obviously, such applications would require the assessment of production trade-offs specific to the area of application.

\section{Problem description}

The described application concerns the analysis of efficiency of wheat-producing agricultural farms in different regions of Turkey. The data for this study is obtained from the Farm Accountancy Data Network (FADN) held by the Turkish Ministry of Agriculture.

FADN is the agricultural data network project established and defined by the European Union (EC, 2010). The data in the network consist of physical and structural data, as well as economic and financial data, collected through annual surveys carried out by the member states. In general, data are collected from "commercial farms". A commercial farm is defined as "a farm, which is large enough to provide a main activity for the farmer and a level of income sufficient to support his or her family. In practical terms, in order to be classified as commercial, a farm must exceed a minimum economic size" (EC, 2010). As a candidate country for the European Union, Turkey started a project for establishing a FADN in 2007. For this purpose, the Turkish Ministry of Agriculture initiated a pilot data collection in 2009.

\subsection{The data set}

The FADN data set provided to us by the Turkish Ministry of Agriculture consisted of 374 commercial farms, with the data collected in 2009. In 2002, to shape agricultural policies and practices, the agricultural policy makers in Turkey divided the country into 12 regions depending on several factors. Every region consisted of several provinces (Saçl1, 2009). In the pilot FADN data provided to us, 9 regions out of 12 were covered. The Ministry selected one representative province in each region and collected data from the commercial farms located in the selected provinces. 
Table 1 shows the regions and their representative provinces, indicated by the main city in the province, together with the corresponding number of farms recorded in FADN. The missing data are identified by symbol "-".

Because wheat is the most common crop cultivated by the majority of farms in the data set and in order to simplify the exposition of our method, we limit our study to wheatproducing farms only. This reduces our sample to 249 wheat-producing farms in all regions in total. We further exclude the farms that have missing or obviously erroneous data in FADN. Because the East Black Sea region includes only 3 farms producing wheat, this region is also excluded from our analysis, resulting in 8 regions in our final sample. All this reduces the number of wheat-producing farms to 213 in total.

Table 1. Sample sizes and number of crop types for each region in Turkish FADN

\begin{tabular}{|c|c|c|c|c|c|c|}
\hline & Region & City & $\begin{array}{c}\text { Number of } \\
\text { farms in } \\
\text { FADN }\end{array}$ & $\begin{array}{l}\text { Number of wheat- } \\
\text { producing farms }\end{array}$ & $\begin{array}{c}\text { Final } \\
\text { sample } \\
\text { size }\end{array}$ & $\begin{array}{c}\text { Number } \\
\text { of crop } \\
\text { types }\end{array}$ \\
\hline 1 & Istanbul & Istanbul & - & - & - & - \\
\hline 2 & West Marmara & Tekirdag & 45 & 44 & 39 & 14 \\
\hline 3 & Aegean & Izmir & 45 & 19 & 16 & 17 \\
\hline 4 & East Marmara & Bursa & 46 & 28 & 27 & 20 \\
\hline 5 & West Anatolia & Konya & 43 & 35 & 35 & 17 \\
\hline 6 & Mediterranean & Adana & 45 & 35 & 30 & 16 \\
\hline 7 & Middle Anatolia & Nevsehir & 35 & 31 & 26 & 15 \\
\hline 8 & West Black Sea & Samsun & - & - & - & - \\
\hline 9 & East Black Sea & Giresun & 35 & 3 & - & - \\
\hline 10 & North East Anatolia & Erzurum & 30 & 19 & 14 & 10 \\
\hline 11 & Middle East Anatolia & Malatya & - & - & - & - \\
\hline \multirow[t]{2}{*}{12} & South East Anatolia & Sanliurfa & 50 & 35 & 26 & 10 \\
\hline & & Total & 374 & 249 & 213 & \\
\hline
\end{tabular}

From the last column of Table 1 we observe that, in every region, the wheatproducing farms cultivate from 10 to 20 types of different crops, including wheat.

In our study, we compare the efficiency of farms within each region separately. Relying on the regional classification by the Turkish Ministry of Agriculture implemented in FADN, we assume that the wheat-producing farms in the each region are sufficiently homogeneous in terms of environmental factors that include geography, soil quality, weather and socio-economic characteristics. This allows us to regard all wheat-producing farms in the 
same region as operating in the same production technology. The fact that most farms produce only a subset of all available crops is not considered as evidence pointing to different technologies: it is assumed that the farms choose to cultivate only a subset of crops. This assumption is in line with many other studies based on FADN data where it is assumed that crop-producing farms (including wheat-producing farms) in the same region operate in the same production technology (see, e.g., Oude Lansink et al. 2002 and Skevas et al. 2012).

\subsection{The outputs}

The outputs in our models (constructed for each region separately) include all individual crops produced by at least one farm in the region and measured in tonnes (1 tonne $=1000 \mathrm{~kg}$ ). Overall, there are 36 different crops, including wheat. The largest number of crops is cultivated in East Marmara region - this includes 20 different crops.

Table 2 summarises the crop types in the final sample and the number of farms producing them in each region. In all regions, every farm produces wheat and some other additional crops. Some of the latter are cultivated by the majority of farms, and some are produced only by a limited number of farms, often by just one farm. We observe a high level of non-homogeneity in terms of production specialisation between the farms in the same region - this makes it a good candidate for the methodology proposed in Section 3.

\subsection{The inputs}

The FADN data set provided to us consists of more than 2000 items (variables). Not all of these are of interest for our application. Many of these items represent aggregated values, often in an overlapping fashion. In addition, not all of them are complete. An official document explaining the meaning and content of each item was provided to us together with the data set (EC, 2009). We rely on these descriptions when identifying the inputs for our DEA models.

Overall we use four inputs in our DEA models. These reflect the data available to us and are consistent with the literature. Below we briefly comment on the choice of inputs.

Land is defined as the utilized agricultural area and is used as an input in almost every study of farm efficiency. In our data set, the utilised areas for all crops are added together to obtain the total land use for each farm, expressed in decares $\left(1\right.$ decare $\left.=1000 \mathrm{~m}^{2}\right)$.

Labour may be expressed as labour costs (Färe et al., 1997), annual work units (Latruffe et al., 2004; Davidova and Latruffe, 2007) or labour hours (Reinhard et al., 2000; Iráizoz et al., 2003; Luik et al., 2009). In our models we use labour costs as reported in the 
Turkish FADN and measured in Turkish Lira (TL). These costs are the sum of gross wages and in-kind payments paid to the employees. The in-kind payments are made in the form of rent, farm products, meals, lodging, and so on (EC, 2009). 
Table 2. Crop types and the corresponding number of farms in each region

\begin{tabular}{|c|c|c|c|c|c|c|c|c|}
\hline We & t Marmara & $\begin{array}{l}\text { Number } \\
\text { of farms }\end{array}$ & Aeg & ean & $\begin{array}{l}\text { Number } \\
\text { of farms }\end{array}$ & Ea: & Marmara & $\begin{array}{l}\text { Number } \\
\text { of farms }\end{array}$ \\
\hline 1 & Wheat & 39 & 1 & Wheat & 16 & 1 & Wheat & 27 \\
\hline 2 & Sunflower & 38 & 2 & Fodder maize & 12 & 2 & Grain maize & 17 \\
\hline 3 & Barley & 15 & 3 & Grain maize & 9 & 3 & Fodder maize & 15 \\
\hline 4 & Oilseed rape & 14 & 4 & Vetch & 6 & 4 & Tomatoes & 15 \\
\hline 5 & Grain maize & 10 & 5 & Lucerne & 5 & 5 & Sugar beet & 10 \\
\hline 6 & Vetch & 10 & 6 & Cotton & 4 & 6 & Lucerne & 8 \\
\hline 7 & Fodder maize & 5 & 7 & Barley & 4 & 7 & Peas & 8 \\
\hline 8 & Watermelon & 4 & 8 & Olives for olive oil & 3 & 8 & Vetch & 8 \\
\hline 9 & Lucerne & 3 & 9 & Table olives & 3 & 9 & Sunflower & 5 \\
\hline 10 & Sugar beet & 3 & 10 & Pepper & 3 & 10 & Oats & 4 \\
\hline 11 & Onions & 3 & 11 & Tomatoes & 3 & 11 & Barley & 4 \\
\hline 12 & Oats & 2 & 12 & Grapes for wine & 2 & 12 & Pepper & 4 \\
\hline 13 & Grass & 2 & 13 & Oats & 2 & 13 & Table olives & 4 \\
\hline 14 & Grapes for wine & 2 & 14 & Tobacco & 2 & 14 & Onions & 3 \\
\hline & & & 15 & Aubergine & 1 & 15 & Beans & 2 \\
\hline & & & 16 & Potatoes & 1 & 16 & Watermelon & 2 \\
\hline & & & 17 & Watermelon & 1 & 17 & Cherry & 1 \\
\hline & & & & & & 18 & Melon & 1 \\
\hline & & & & & & 19 & Pear & 1 \\
\hline & & & & & & 20 & Potatoes & 1 \\
\hline We & t Anatolia & $\begin{array}{l}\text { Number } \\
\text { of farms }\end{array}$ & Me & diterranean & $\begin{array}{l}\text { Number } \\
\text { of farms }\end{array}$ & Mi & dle Anatolia & $\begin{array}{l}\text { Number } \\
\text { of farms }\end{array}$ \\
\hline 1 & Wheat & 35 & 1 & Wheat & 30 & 1 & Wheat & 26 \\
\hline 2 & Barley & 22 & 2 & Grain maize & 21 & 2 & Barley & 19 \\
\hline 3 & Sugar beet & 21 & 3 & Sunflower & 10 & 3 & Table grapes & 12 \\
\hline 4 & Lucerne & 9 & 4 & Cotton & 7 & 4 & Vetch & 10 \\
\hline 5 & Beans & 7 & 5 & Barley & 6 & 5 & Potatoes & 6 \\
\hline 6 & Fodder maize & 7 & 6 & Oranges & 4 & 6 & Rye & 6 \\
\hline 7 & Sunflower & 7 & 7 & Fodder maize & 3 & 7 & Grain maize & 6 \\
\hline 8 & Vetch & 7 & 8 & Watermelon & 2 & 8 & Lucerne & 5 \\
\hline 9 & Peas & 5 & 9 & Nuts & 2 & 9 & Courgette seed & 4 \\
\hline 10 & Grain maize & 3 & 10 & Lemons & 1 & 10 & Grapes for wine & 4 \\
\hline 11 & Oats & 3 & 11 & Table olives & 1 & 11 & Peas & 4 \\
\hline 12 & Potatoes & 3 & 12 & Vetch & 1 & 12 & Oats & 3 \\
\hline 13 & Apple & 2 & 13 & Lucerne & 1 & 13 & Apple & 3 \\
\hline 14 & Cherry & 2 & 14 & Oilseed rape & 1 & 14 & Sugar beet & 2 \\
\hline 15 & Grass & 1 & 15 & Tomatoes & 1 & 15 & Sunflower & 1 \\
\hline 16 & Lentil & 1 & 16 & Onions & 1 & & & \\
\hline 17 & Rye & 1 & & & & & & \\
\hline Nor & th East Anatolia & $\begin{array}{l}\text { Number } \\
\text { of farms }\end{array}$ & Sou & th East Anatolia & $\begin{array}{l}\text { Number } \\
\text { of farms }\end{array}$ & & & \\
\hline 1 & Wheat & 14 & 1 & Wheat & 26 & & & \\
\hline 2 & Lucerne & 14 & 2 & Grain maize & 17 & & & \\
\hline 3 & Grass & 9 & 3 & Cotton & 13 & & & \\
\hline 4 & Vetch & 8 & 4 & Barley & 4 & & & \\
\hline 5 & Barley & 8 & 5 & Nuts & 2 & & & \\
\hline 6 & Potatoes & 4 & 6 & Lentil & 2 & & & \\
\hline 7 & Grain maize & 3 & 7 & Fodder maize & 2 & & & \\
\hline 8 & Sugar beet & 3 & 8 & Tomatoes & 1 & & & \\
\hline 9 & Rye & 1 & 9 & Pepper & 1 & & & \\
\hline 10 & Sunflower & 1 & 10 & Aubergine & 1 & & & \\
\hline
\end{tabular}


Crop production costs are among the key factors considered in all studies, but their exact definition varies between different studies. Costs may be aggregated into cultivation costs (Iráizoz et al., 2003), intermediate consumption (Latruffe et al., 2004; Davidova and Latruffe, 2007; Luik et al., 2009), materials (Alene et al., 2006), total expenses (Amores and Contreras, 2009), or variable inputs (Reinhard et al., 2000; Cherchye and Van Puyenbroeck, 2007). These aggregated variables represent the sum of costs of various items used in agricultural production such as energy, fertilizer, feed, fuel, seed, machinery, pesticides, water and farming overheads.

In our application, we use the crop production costs as the sum of the following costs included in FADN and measured in Turkish Lira: seeds and seedlings purchased or produced on the farm, purchased fertilizers and soil improvers, crop protection products and other specific crop costs. We specifically exclude the costs spent on the maintenance of the farm these are used in the definition the last input described below.

Capital expenditures used as an input in our model is a variant of the capital variable used in the literature. In different studies the definition of the capital input has been based, with some variations, on the book value of machinery and buildings (Brümmer, 2001; Oude Lansink et al., 2002; Iráizoz et al., 2003; Skevas et al., 2012), depreciated value of total assets (Latruffe et al., 2004; Davidova and Latruffe, 2007; Luik et al., 2009), and the annual capital costs (Färe et al., 1997). Based on the available data, we define the capital input in our model as capital expenditures expressed in Turkish Lira. This consists of three main types of cost: machinery costs, farming overheads and land charges, and is close to the annual capital costs used by Färe et al. (1997).

\section{Methodology}

The suggested approach to the efficiency assessment of agricultural farms with nonhomogeneous production profiles is based on the notion of production trade-offs (Podinovski, 2004). Such trade-offs represent simultaneous changes to the inputs and outputs that are assumed technologically possible if applied to any DMU in the given technology. Production trade-offs are incorporated as additional terms in the envelopment VRS and CRS DEA models. They correspond to weight restrictions in the dual multiplier model.

Before the formal introduction of this methodology, we consider a motivational example and illustrate the meaning of production trade-offs in the context of evaluation of farm efficiency. 


\subsection{Motivational example}

Consider the four farms: $A, B, C$, and $D$, as shown in Table 3. Each farm uses the same area of land (the measurement units for land are not important for our example) to produce two outputs: wheat and barley, measured in tonnes. Two farms, $B$ and $C$, produce both wheat and barley. Farms $A$ and $B$ have specialised production profiles: farm $A$ produces only barley, and farm $D$ produces only wheat.

Table 3. Farms in the example

\begin{tabular}{cccc}
\hline Farm & Land & Wheat & Barley \\
\hline$A$ & 1 & 0 & 4.5 \\
$B$ & 1 & 2 & 4 \\
$C$ & 1 & 4 & 3 \\
$D$ & 1 & 5 & 0 \\
\hline
\end{tabular}

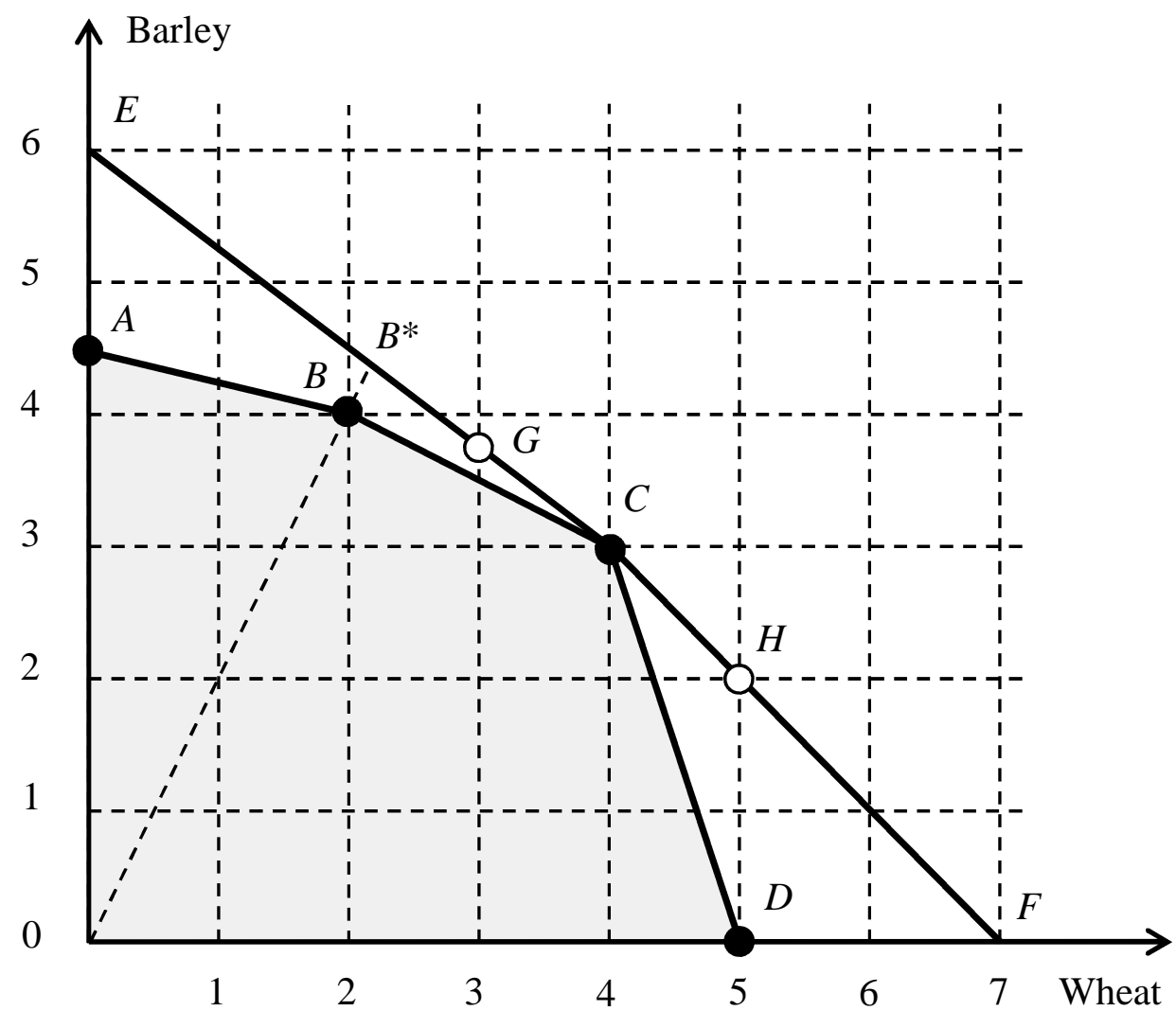

Figure 1. Technology with production trade-offs

The shaded area in Figure 1 represents the section of the conventional VRS or CRS technology that corresponds to the level of land input of the four farms. (This section does 
not depend on whether we assume VRS or CRS.) The efficient frontier is represented by the broken line $A B C D$, and all observed farms are therefore efficient.

Observe that farms $A$ and $B$ produce much less of wheat than farm $C$. However, because they produce a relatively small additional amount of barley, both farms $A$ and $B$ are efficient. Similarly, farm $D$ produces no barley (a reduction of three tonnes compared to farm $C)$ but is efficient because it produces one additional tonne of wheat.

The fact that all four farms in Figure 1 are efficient is in conflict with the fact that wheat and barley are similar crops, and a reduction in the production of one of these needs to be compensated by an increase of a similar (though not identical) volume of the other crop ${ }^{3}$.

We deal with the above problem by asking experts to assess the relative difficulty of the production of wheat and barley (in terms of consumption of resources), taking into account possible variations between the farms in the region (in which farms $A, B, C$ and $D$ are located). The following two statements are based on the actual judgements elicited in the application discussed in Section 4.

Judgement 1. Any farm in the region can produce at least 0.75 tonnes of barley instead of 1 tonne of wheat, without claiming additional resources.

Judgement 2. Any farm in the region can produce at least 1 tonne of wheat instead of 1 tonne of barley, without claiming additional resources.

The above two judgements are examples of production trade-offs that are formally defined in the next section. Both reflect the fact that the production of barley may require slightly more resources than wheat. Depending on the farm, the parity in the use of resources is within the range from 0.75 tonnes to 1 tonne of barley for 1 tonne of wheat. Therefore, it is safe to assume that, if the production of wheat is reduced by 1 tonne, the released resources are sufficient to produce at least 0.75 tonnes of barley, at any farm in the region - this is stated in Judgement 1. Similarly, in order to increase the production of wheat by 1 tonne, it suffices to release the resources by reducing the production of barley by 1 tonne - this is stated in Judgement 2.

In order to incorporate the above two judgements in the model of technology, refer to Figure 1 and consider, for example, farm $C$. Using Judgement 1 , we can reduce the

\footnotetext{
${ }^{3}$ In the dual space the slope of the efficient frontier $C D$ is excessively steep and means that the shadow price of wheat on this part of frontier is significantly higher than the shadow price of barley, which is unrealistic. Similarly, the two slopes of the parts of the efficient frontier $A B$ and $B C$ are excessively flat and imply that, on these parts of the frontier, the shadow price of barley is significantly higher than that of wheat, which is also unacceptable.
} 
production of wheat at farm $C$ by 1 tonne and simultaneously increase the production of barley by 0.75 tonnes. This creates the hypothetical DMU $G$ which is assumed to be producible and must be in the technology. (Clearly, we should also include all points between $C$ and $G$ that correspond to the reduction of wheat production by less than 1 tonne.) Continuing the process of substitution of 1 tonne of wheat by 0.75 tonnes of barley, we add the line $C E$ to the technology.

Similarly, starting at farm $C$ and employing Judgement 2, we can increase the production of wheat by 1 tonne and simultaneously reduce the production of barley by 1 tonne. This creates farm $H$. Repeating the process, we add the line $C F$ to the technology.

Based on Judgements 1 and 2, we have expanded the technology to the quadrangle $E C F O$. Note that, in principle, we should repeat the above procedure starting at all other points in the technology, including farms $A, B$ and $C$. However, this does not create new DMUs because all the resulting farms are under the lines ECF and are, therefore, already included in the technology.

Note that the new efficient frontier is the line $E C F$, and only farm $C$ remains efficient. This means that the discrimination of the new model that incorporates Judgements 1 and 2 is significantly better than that of the original model. Also note that the radial projection of farm $B$ on the new efficient frontier (point $B^{*}$ ) is technologically feasible (producible). This is because $B^{*}$ is obtained from $C$ by an application of Judgement 1. Consequently, the output radial efficiency of farm $B$ (equal to the ratio of $O B$ to $O B^{*}$ ) is a technologically realistic improvement factor. The same observation is true for the other two farms, $A$ and $D$.

\subsection{DEA models with production trade-offs}

Let us show how Judgements 1 and 2 from the above example, and other similar statements, can be incorporated in a formulation of a DEA model. Consider the set of $n$ observed DMUs that operate in a technology with $m$ inputs and $s$ outputs. Observed DMUs are denoted $\left(X_{j}, Y_{j}\right), j=1,2, \ldots, n$, where vectors $X_{j} \in \mathbb{R}_{+}^{m}$ and $Y_{j} \in \mathbb{R}_{+}^{S}$. The unit whose efficiency is being assessed is denoted $\left(X_{0}, Y_{0}\right)$.

Following Podinovski (2004), production trade-offs are stated in the form of pairs of vectors $(P, Q)$. The components of vectors $P \in \mathbb{R}^{m}$ and $Q \in \mathbb{R}^{s}$ can be positive, negative or zero, and indicate simultaneous changes to the corresponding inputs and outputs that are assumed possible at any unit in the technology. 
Using this notation, Judgements 1 and 2 from the above example are stated as the following production trade-offs $\left(P_{1}, Q_{1}\right)$ and $\left(P_{2}, Q_{2}\right)$ :

$$
\begin{aligned}
& P_{1}=\mathbf{0}, \quad Q_{1}=(-1 ; 0.75)^{T} \\
& P_{2}=\mathbf{0}, \quad Q_{2}=(1 ;-1)^{T}
\end{aligned}
$$

where $\mathbf{0}$ is the zero input vector and the sign ${ }^{T}$ denotes transposition.

Suppose we have identified $K$ different production trade-offs ${ }^{4}$

$$
\left(P_{t}, Q_{t}\right), t=1,2, \ldots, K
$$

Following Podinovski (2004), the output-oriented CRS model that incorporates tradeoffs (3) is stated as follows:

$\operatorname{Max} \theta$

$$
\begin{aligned}
& \text { s.t } \sum_{j=1}^{n} \lambda_{j} Y_{j}+\sum_{t=1}^{K} \pi_{t} Q_{t} \geq \theta Y_{0} \\
& \sum_{j=1}^{n} \lambda_{j} X_{j}+\sum_{t=1}^{K} \pi_{t} P_{t} \leq X_{0} \\
& \lambda, \pi \geq 0, \theta \text { sign free }
\end{aligned}
$$

In model (4), $\lambda \in \mathbb{R}_{+}^{n}$ is the intensity vector, and $\pi \in \mathbb{R}_{+}^{K}$ is the vector that represents the proportions $\pi_{t}$ in which the trade-offs $\left(P_{t}, Q_{t}\right)$ are applied. The meaning of the terms on the left-hand side of model (4) is clear: the first group of terms (with multipliers $\lambda_{j}$ ) generates a unit in the standard CRS technology, and the trade-offs $\left(P_{t}, Q_{t}\right)$ are subsequently used (in proportions $\pi_{t}$ ) to modify this unit.

The VRS variant of model (4) is obtained by the incorporation of the normalising equality $\mathbf{1} \lambda=1$, where $1 \in \mathbb{R}^{n}$ is the vector of ones. The input-oriented DEA models are

\footnotetext{
${ }^{4}$ In the described application to agricultural farms vectors $P_{t}$ are zero vectors. This is because each trade-off (3) involves changes only to the outputs, and no inputs are modified. In some applications it may be possible to specify a production trade-off involving two inputs (for example, between labour and costs). The corresponding vector $P_{t}$ would then reflect the changes, and $Q_{t}$ would be a zero vector. It may also be possible to specify more complex trade-offs, involving (several) inputs and outputs simultaneously. An example of this in the context of higher education is given in Podinovski (2007a).
} 
obtained in the usual way: by attaching the improvement factor $\theta$ to the vector $X_{0}$ (instead of $Y_{0}$ ) on the right-hand side of program (4) and changing its objective function to the minimization of $\theta$.

The dual programs to model (4) and its VRS variant are the standard CRS (respectively, VRS) output-oriented multiplier models that incorporate the following additional weight restrictions induced by trade-offs (3):

$$
u^{T} Q_{t}-v^{T} P_{t} \leq 0, \quad t=1,2, \ldots, K .
$$

For example, the production trade-off (1) in the envelopment model is transformed to the weight restriction $-u_{1}+0.75 u_{2} \leq 0$ in the corresponding multiplier model. Similarly, the trade-off (2) translates to the weight restriction $u_{1}-u_{2} \leq 0$. This correspondence is illustrated in Appendix in which we present DEA models for the example considered in Section 3.1.

\section{Application}

Below we demonstrate an application of the described methodology to the analysis of efficiency of agricultural farms in different regions of Turkey. The background of this application and definition of inputs and outputs were given in Section 2.

\subsection{Identification of production trade-offs}

The described methodology is based on the use of production trade-offs between different crops. Theoretically, the trade-offs can be specified between any pairs of crops (for example, between oats and peas) and, more generally, between any subsets of inputs and/or outputs (Podinovski, 2004). In order to keep the application simple and taking into account that wheat is the principle crop produced by all farms in our samples, we identified the tradeoffs only between wheat and one other crop at a time ${ }^{5}$.

We collected expert opinion about production trade-offs from two sources of expertise. The main source reflected the practitioner point of view - production trade-offs between wheat and other crops were provided to us by agricultural engineers working as consultants at a local chamber of agriculture in Turkey. As a secondary source used to verify

\footnotetext{
${ }^{5}$ Keeping the structure of production trade-offs simple also helps to avoid a possible inconsistency in expert judgements. The use of more complex trade-offs increases the chances of inconsistency that manifests itself in an infeasible DEA multiplier model and other problematic outcomes. In such cases, a test of consistency generally needs to be performed (Podinovski and Bouzdine-Chameeva, 2013).
} 
the opinion obtained from the main source and to obtain a few missing trade-offs, we used our academic contacts at an agricultural department of a Turkish university.

When designing the questions to be put to the experts, we felt that it would be difficult and unnecessary to explain the exact mathematical meaning of production trade-offs within the DEA approach. Instead, we decided to ask the questions in a simplified way that would be intuitively meaningful to a person with agricultural expertise, and also allow us to translate the answers to production trade-offs.

We started our discussions with experts by explaining the purpose of our analysis and outlining the input and output measures used in it. We then verified that we were in agreement that some crops required more resources per unit of output than the other. We then proceeded with the main questions that required the comparison of resources used to produce wheat and the other crops. Our discussion with the experts continued along the following lines. In the case of barley we asked:

- Approximately, how much of barley (in tonnes) could be produced with the same resources (inputs) that are needed for the production of 1 tonne of wheat?

We did not require a precise answer to the above question. Instead, we expected an answer in the form of a range between the lower and upper bounds that would account for different working practices of different farms and their locations. We also needed to take into account the level of certainty with which the experts could answer the above question.

Three ranges corresponding to different levels of certainty were obtained as shown in Table 4, in the row corresponding to barley.

Wide range $[0.75 ; 1]$ represented the highest level of confidence of experts that the resources required for the production of 1 tonne of wheat were sufficient for the production between 0.75 and 1 tonne of barley, at any farm in the region.

Medium range $[0.8 ; 0.95]$ represented the less certain judgement that most farms in the region should probably be able to produce the amount of barley in the specified reduced range, instead of 1 tonne of wheat.

Narrow range $[0.85 ; 0.9]$ was the least certain judgement that would apply to a typical farm only.

Because of the highest confidence of experts in the Wide range, we used it in the main part of our analysis. The Medium and Narrow bounds were also used but only to investigate 
if the tightening of the bounds (at the expense of their reliability) gave a sufficient improvement in efficiency discrimination to justify the reduced confidence in the resulting model. The latter is discussed in Section 4.3.

Table 4. Trade-off ranges (lower and upper bounds)

\begin{tabular}{|c|c|c|c|c|c|c|c|}
\hline & \multirow{2}{*}{ Crops } & \multicolumn{2}{|c|}{ Wide range } & \multicolumn{2}{|c|}{ Medium range } & \multicolumn{2}{|c|}{ Narrow range } \\
\hline & & Lower & Upper & Lower & Upper & Lower & Upper \\
\hline & Cereals & & & & & & \\
\hline 1 & Grain maize & 2 & 3 & 2.2 & 2.8 & 2.4 & 2.6 \\
\hline 2 & Barley & 0.75 & 1 & 0.8 & 0.95 & 0.85 & 0.9 \\
\hline 3 & Oats & 0.25 & 0.4 & 0.275 & 0.375 & 0.3 & 0.35 \\
\hline 4 & Rye & 0.5 & 0.8 & 0.55 & 0.75 & 0.6 & 0.7 \\
\hline \multirow[t]{2}{*}{5} & Triticale & 0.6 & 0.9 & 0.65 & 0.85 & 0.7 & 0.8 \\
\hline & Fodder crops & & & & & & \\
\hline 6 & Lucerne & 1.2 & 6 & 1.3 & 5.9 & 1.5 & 5.5 \\
\hline 7 & Fodder maize & 10 & 20 & 12 & 18 & 14 & 16 \\
\hline \multirow[t]{2}{*}{8} & Grass & - & - & - & - & - & - \\
\hline & Field crops & & & & & & \\
\hline 9 & Vetch & 0.3 & 0.6 & 0.35 & 0.55 & 0.4 & 0.5 \\
\hline 10 & Peas & 0.2 & 0.4 & 0.25 & 0.35 & 0.275 & 0.325 \\
\hline 11 & Beans & 0.25 & 0.4 & 0.275 & 0.375 & 0.3 & 0.35 \\
\hline 12 & Lentil & 0.15 & 0.3 & 0.175 & 0.275 & 0.2 & 0.25 \\
\hline 13 & Sunflower & 0.4 & 0.9 & 0.5 & 0.8 & 0.6 & 0.7 \\
\hline 14 & Oilseed rape & 0.5 & 1 & 0.6 & 0.9 & 0.7 & 0.8 \\
\hline 15 & Cotton & 0.8 & 1.4 & 0.9 & 1.3 & 1 & 1.2 \\
\hline 16 & Potatoes & 4 & 7 & 4.5 & 6.5 & 5 & 6 \\
\hline 17 & Sugar beet & 13 & 20 & 14 & 19 & 15 & 18 \\
\hline \multirow[t]{2}{*}{18} & Tobacco & 0.4 & 0.9 & 0.5 & 0.8 & 0.6 & 0.7 \\
\hline & Permanent crops & & & & & & \\
\hline 19 & Oranges & 0.6 & 1.2 & 0.7 & 1.1 & 0.8 & 1 \\
\hline 20 & Lemons & 0.45 & 0.9 & 0.45 & 0.75 & 0.5 & 0.7 \\
\hline 21 & Apple & 1 & 2.5 & 1.25 & 2.25 & 1.5 & 2 \\
\hline 22 & Cherry & 0.5 & 1 & 0.55 & 0.85 & 0.6 & 0.8 \\
\hline 23 & Pear & 0.6 & 1.2 & 0.7 & 1.1 & 0.8 & 1 \\
\hline 24 & Nuts & - & - & - & - & - & - \\
\hline 25 & Table olives & 0.4 & 1 & 0.5 & 0.9 & 0.6 & 0.8 \\
\hline 26 & Olives for olive oil & 0.4 & 1 & 0.5 & 0.9 & 0.6 & 0.8 \\
\hline 27 & Table grapes & 1.5 & 2.5 & 1.6 & 2.4 & 1.7 & 2.2 \\
\hline \multirow[t]{2}{*}{28} & Grapes for wine & 1.5 & 2.5 & 1.6 & 2.4 & 1.7 & 2.2 \\
\hline & $\begin{array}{l}\text { Vegetables and } \\
\text { non-perennial fruits }\end{array}$ & & & & & & \\
\hline 29 & Watermelon & 4.5 & 6.5 & 4.75 & 6.25 & 5 & 6 \\
\hline 30 & Melon & 3.5 & 5 & 3.75 & 4.75 & 4 & 4.5 \\
\hline 31 & Tomatoes & 4 & 14 & 6 & 12 & 8 & 10 \\
\hline 32 & Pepper & 3 & 6 & 3.5 & 5.5 & 4 & 5 \\
\hline 33 & Courgette seed & 0.175 & 0.3 & 0.175 & 0.275 & 0.2 & 0.25 \\
\hline 34 & Aubergine & 2.5 & 4 & 2.75 & 3.75 & 3 & 3.5 \\
\hline 35 & Onion & 0.8 & 1.5 & 0.9 & 1.3 & 1 & 1.2 \\
\hline
\end{tabular}

To verify that the Wide bounds were sufficiently wide (to apply to all farms and all reasonable production conditions), we obtained an additional confirmation from the experts that the following statement was correct: 
- In all normal circumstances and at all farms, the resources required for the production of 1 tonne of wheat are definitely sufficient for the production of at least 0.75 tonnes of barley, but are definitely insufficient for the production of more than 1 tonne of barley.

We subsequently restated the Wide range for barley in the form of Judgements 1 and 2 presented in Section 3, and converted them to the two corresponding production trade-offs as discussed. The trade-off ranges were also obtained for the other crops, and each range was converted to a pair of trade-offs in a similar way.

Note that the use of the Wide range for the assessment of production trade-offs complies with the theoretical requirement (Podinovski, 2004) that the trade-offs should be applicable to any unit in the technology (in our case all farms in the regions of Turkey) ${ }^{6}$. The use of the trade-offs based on the Medium range may not satisfy this requirement, and the use of the Narrow range almost certainly does not.

Table 4 shows the three trade-off ranges obtained by the described procedure: Wide, Medium and Narrow, in the form of their lower and upper bounds. The crops are presented and categorised into five main classes in accordance with the classification adopted by Community Committee for FADN (EC, 2009). Table 4 provides trade-offs for all the crop types in our data set except for grass (produced by some farms in West Marmara, West Anatolia, and North East Anatolia regions) and nuts (produced by some farms in Mediterranean and South East Anatolia regions). For these crops we did not employ any trade-offs in our models.

\subsection{Efficiency evaluation with and without production trade-offs}

We performed the analysis of farm efficiency in each of the eight regions of Turkey separately. The regional classification of the Ministry of Agriculture implied that all farms in the same region could be assumed to operate in a sufficiently similar environment. As discussed in Section 2, for each region we employed four inputs and a large number of outputs that represented all crops produced by the farms in the region. Considering the available sample sizes, the number of outputs in each region appeared to be problematic even before the analysis was performed. For example, in Aegean region, there were 16 farms and

\footnotetext{
${ }^{6}$ To see the importance of this requirement, assume that we are not certain that Judgements 1 and 2, and the trade-offs based on them, apply to all farms. In this case we cannot be certain that these are true at farm $C$ in Figure 1. Therefore we should have little confidence in the efficient frontier $E C F$ induced by the two trade-offs, and in the resulting efficiency scores.
} 
17 outputs. It was clear that the discrimination of the standard DEA models would be unsatisfactory.

We used the output-oriented DEA models under both the VRS and CRS assumptions. The computations were performed using General Algebraic Modeling System (GAMS). Table 5 shows the summary of results by region. As expected, the conventional DEA models (without production trade-offs) did not discriminate well because of the large number of outputs and inputs included in the models. For example, in East Marmara region, in which 27 farms produced 20 different crops, all farms were shown as efficient in both VRS and CRS models. In the other regions the CRS models showed a generally better discrimination than the VRS models.

The results changed significantly after we incorporated the production trade-offs based on the Wide range in Table 4. For the actual computations, we used the multiplier forms of model (4), in which all the trade-offs (3) were transformed to weight restrictions (5). For example, in the DEA models for East Marmara region, we used 38 weight restrictions: these represented the lower and upper bounds on the production trade-offs obtained for 19 crops cultivated in the region.

As seen from the corresponding columns of Table 5, the incorporation of production trade-offs from the Wide ranges generally significantly improved the discrimination of both VRS and CRS DEA models. In particular, the average efficiency scores and the proportion of efficient and inefficient farms in Mediterranean region changed dramatically.

For regions such as Aegean, West Marmara and South East Anatolia, even though the average efficiency level remained high, the models with production trade-offs were still able to identify more inefficient farms.

\subsection{Sensitivity of results with respect to trade-off ranges}

In addition to the main part of our analysis in which we used the Wide ranges to construct production trade-offs, we also performed analysis using the Medium and Narrow ranges, as shown in Table 4. The results are shown in the corresponding columns of Table 5.

As expected, the use of the Medium and Narrow ranges further (compared to the use of Wide ranges) improved the discrimination of DEA models for most regions, and under both VRS and CRS assumptions. Note that this improvement was, for most regions, less significant than the improvement achieved by the use of the Wide trade-off range in comparison to the standard DEA models without trade-offs. One can argue that it is not worth sacrificing the credibility of the model (by using less certain expert judgements) for the 
relatively minor gains in efficiency discrimination, especially when the most reliable Wide range produces more than satisfactory efficiency discrimination in the first place. 
Table 5. Summary of efficiency analysis with different trade-off ranges

\begin{tabular}{|c|c|c|c|c|c|c|c|c|c|}
\hline \multirow[b]{2}{*}{ Region } & & \multicolumn{4}{|l|}{ VRS model } & \multicolumn{4}{|l|}{ CRS model } \\
\hline & & $\begin{array}{l}\text { No } \\
\text { trade-offs }\end{array}$ & $\begin{array}{l}\text { Wide } \\
\text { range } \\
\text { trade-offs }\end{array}$ & $\begin{array}{l}\text { Medium } \\
\text { range } \\
\text { trade-offs }\end{array}$ & $\begin{array}{l}\text { Narrow } \\
\text { range } \\
\text { trade-offs }\end{array}$ & $\begin{array}{l}\text { No } \\
\text { trade-offs }\end{array}$ & $\begin{array}{l}\text { Wide } \\
\text { range } \\
\text { trade-offs }\end{array}$ & $\begin{array}{l}\text { Medium } \\
\text { range } \\
\text { trade-offs }\end{array}$ & $\begin{array}{l}\text { Narrow } \\
\text { range } \\
\text { trade-offs }\end{array}$ \\
\hline \multirow{3}{*}{ West Marmara } & Average efficiency & 0.98 & 0.96 & 0.95 & 0.92 & 0.97 & 0.92 & 0.88 & 0.84 \\
\hline & Efficient farms & 35 & 24 & 20 & 15 & 30 & 12 & 9 & 8 \\
\hline & Inefficient farms & 4 & 15 & 19 & 24 & 9 & 27 & 30 & 31 \\
\hline \multirow{3}{*}{ Aegean } & Average efficiency & 1.00 & 0.98 & 0.98 & 0.97 & 1 & 0.97 & 0.95 & 0.91 \\
\hline & Efficient farms & 16 & 1 & 1 & 3 & 16 & 3 & 4 & 7 \\
\hline & Inefficient farms & 0 & 15 & 15 & 13 & 0 & 13 & 12 & 9 \\
\hline \multirow{3}{*}{ East Marmara } & Average efficiency & 1.00 & 0.74 & 0.69 & 0.63 & 1.00 & 0.70 & 0.63 & 0.56 \\
\hline & Efficient farms & 27 & 10 & 10 & 6 & 27 & 8 & 6 & 5 \\
\hline & Inefficient farms & 0 & 17 & 17 & 21 & 0 & 19 & 21 & 22 \\
\hline \multirow{3}{*}{ West Anatolia } & Average efficiency & 0.98 & 0.86 & 0.84 & 0.81 & 0.94 & 0.72 & 0.69 & 0.65 \\
\hline & Efficient farms & 33 & 19 & 17 & 14 & 25 & 7 & 5 & 5 \\
\hline & Inefficient farms & 2 & 16 & 18 & 21 & 10 & 28 & 30 & 30 \\
\hline \multirow{3}{*}{ Mediterranean } & Average efficiency & 1.00 & 0.51 & 0.47 & 0.44 & 0.99 & 0.24 & 0.22 & 0.21 \\
\hline & Efficient farms & 28 & 8 & 8 & 6 & 26 & 3 & 3 & 3 \\
\hline & Inefficient farms & 2 & 22 & 22 & 24 & 4 & 27 & 27 & 27 \\
\hline \multirow{3}{*}{ Middle Anatolia } & Average efficiency & 0.97 & 0.81 & 0.79 & 0.76 & 0.95 & 0.68 & 0.67 & 0.64 \\
\hline & Efficient farms & 22 & 13 & 13 & 12 & 22 & 8 & 8 & 8 \\
\hline & Inefficient farms & 4 & 13 & 13 & 14 & 4 & 18 & 18 & 18 \\
\hline \multirow{3}{*}{ North East Anatolia } & Average efficiency & 1.00 & 0.97 & 0.96 & 0.95 & 0.97 & 0.89 & 0.89 & 0.87 \\
\hline & Efficient farms & 13 & 11 & 11 & 11 & 12 & 9 & 9 & 8 \\
\hline & Inefficient farms & 1 & 3 & 3 & 3 & 2 & 5 & 5 & 6 \\
\hline \multirow{3}{*}{ South East Anatolia } & Average efficiency & 0.99 & 0.97 & 0.96 & 0.96 & 0.97 & 0.92 & 0.91 & 0.89 \\
\hline & Efficient farms & 24 & 20 & 20 & 18 & 19 & 12 & 10 & 10 \\
\hline & Inefficient farms & 2 & 6 & 6 & 8 & 7 & 14 & 16 & 16 \\
\hline
\end{tabular}


Overall, it appears that the use of the most reliable Wide trade-off ranges represents a good balance between the need to improve the efficiency discrimination while at the same time have confidence in the results. This observation suggests that, in similar practical applications of DEA one should not aim to obtaining as tight trade-off ranges as possible - it would normally suffice to use comfortably wide ranges that one can have full confidence in.

\subsection{Explaining radial targets and reference sets}

In the conventional VRS DEA models the radial targets (projections) of inefficient DMUs represent convex combinations of observed DMUs, or are dominated by such convex combinations. In the CRS models the radial targets are obtained in a similar way, either as conical combinations of observed units, or as units dominated by them. In this section, we discuss how the radial targets of inefficient DMUs can be explained in the DEA models with production trade-offs. This extends our discussion of the radial target $B^{*}$ in the example in Section 3.1.

To be specific, we consider the output-oriented CRS model (4), where production trade-offs (3) are defined according to the Wide ranges shown in Table 4. Using this model, we evaluate the output radial targets of the 35 farms in the region West Anatolia. A typical farm in this region is Farm 19. Its main products are wheat and sugar beet. It also produces relatively small amounts of beans and sunflower. The actual outputs of this farm are shown in the second column of Table $6 .^{7}$

Table 6. The output radial targets and reference set of Farm 19

\begin{tabular}{|c|c|c|c|c|c|c|}
\hline & \multirow{2}{*}{$\begin{array}{c}\text { Actual } \\
\text { outputs of } \\
\text { Farm 19 }\end{array}$} & \multicolumn{2}{|c|}{$\begin{array}{l}\text { Reference set of } \\
\text { Farm } 19\end{array}$} & \multirow{2}{*}{$\tilde{Y}=\sum_{j=1}^{n} \lambda_{j} Y_{j}$} & \multirow{2}{*}{$\tilde{Q}=\sum_{t=1}^{K} \pi_{t} Q_{t}$} & \multirow{2}{*}{$\begin{array}{c}\text { Output radial } \\
\text { target } Y_{19}^{*} \text { of } \\
\text { Farm } 19\end{array}$} \\
\hline & & Farm 28 & Farm 33 & & & \\
\hline Wheat & 126 & 37 & 53 & 135.58 & 15.3 & 150.88 \\
\hline Lucerne & 0 & 0 & 160 & 9.24 & -9.24 & 0 \\
\hline Fodder maize & 0 & 0 & 350 & 20.22 & -20.22 & 0 \\
\hline Vetch & 0 & 0 & 44 & 2.54 & -2.54 & 0 \\
\hline Beans & 12 & 8 & 0 & 28.66 & -14.29 & 14.37 \\
\hline Sunflower & 1 & 0 & 0 & 0 & 1.2 & 1.2 \\
\hline Potatoes & 0 & 0 & 660 & 38.13 & -38.13 & 0 \\
\hline Sugar beet & 380 & 0 & 1200 & 69.33 & 385.7 & 455.03 \\
\hline
\end{tabular}

\footnotetext{
${ }^{7}$ As shown in Table 1, there are 17 different crops produced by all farms in West Anatolia. Table 6 shows only those crops that are produced either by Farm 19 or its reference set consisting of Farms 28 and 33.
} 
Computations show that Farm 19 is inefficient. The optimal value of the corresponding model (4) is $\theta^{*} \approx 1.197$, and the output radial efficiency of Farm 19 is $1 / \theta^{*} \approx 0.84{ }^{8}$ Therefore, vector $Y_{19}^{*}=\theta^{*} Y_{19}$ shown in the last column of Table 6 is the output radial target of Farm 19. This projection does not change the input vector of Farm 19.

The structure of model (4) allows us to explain why the target vector $Y_{19}^{*}$ is technologically feasible. Denote $\hat{Y}_{19}$ the vector obtained by the calculations on the left-hand side of the output inequalities of program (4). To simplify the exposition, let vectors $\underline{Q}_{t}$ and $\bar{Q}_{t}$ represent the trade-offs constructed using the Wide-range lower and, respectively, upper bounds for crop $t$ as shown in Table 4. For example, $\underline{Q}_{2}=(-1 ; 0.75,0, \ldots, 0)^{\top}$ and $\bar{Q}_{2}=(1 ;-1,0, \ldots, 0)^{\top}$ correspond to crop 2 (barley). ${ }^{9}$ This is the extended form of trade-offs (1) and (2) discussed above.

Using vectors $\lambda^{*}$ and $\pi^{*}$ from the optimal solution to (4), vector $\hat{Y}_{19}$ is obtained as follows (only nonzero components are shown and are rounded): ${ }^{10}$

$$
\begin{aligned}
\hat{Y}_{19}= & 3.58 Y_{28}+0.058 Y_{33} \\
& +1.54 \bar{Q}_{6}+1.01 \bar{Q}_{7}+4.24 \bar{Q}_{9}+35.72 \bar{Q}_{11}+2.99 \underline{Q}_{13}+5.45 \bar{Q}_{16}+29.67 \underline{Q}_{17} .
\end{aligned}
$$

Formula (6) shows that Farms 28 and 33 constitute the reference set for Farm 19 and are combined with the intensity coefficients $\lambda_{28}=3.58$ and $\lambda_{33}=0.058 .{ }^{11}$ Denote the

\footnotetext{
${ }^{8}$ In the standard CRS model the output (and input) radial efficiency of Farm 19 is equal to 0.97.

${ }^{9}$ The dimension of each vector $\underline{Q}_{t}$ and $\bar{Q}_{t}$ is equal to 36 . Components of these vectors correspond to wheat and 35 specialist crops shown in Table 4 . For crops 8 and 24 the trade-offs are not specified. To keep the numbering of crops unchanged, we formally define vectors $\underline{Q}_{t}$ and $\bar{Q}_{t}$ for $t=8$ and 24 as zero vectors. Because inputs are not affected by trade-offs in Table 4 , for all 35 crops the corresponding vectors $\underline{P}_{t}$ and $\bar{P}_{t}$ in formula (3) are zero vectors.

${ }^{10}$ As in the standard CRS and VRS models, the optimal vectors $\lambda^{*}$ and $\pi^{*}$ in program (4) and the vector $\hat{Y}_{19}$ based on them may not be unique.

${ }^{11}$ Both Farms 29 and 33 are efficient in model (4). Generally, program (4) may have alternative optimal solutions, and it is possible that in rare cases of primarily academic interest some of the DMUs corresponding to strictly positive $\lambda_{j}$ are inefficient. However, there always exists an optimal solution to (4) such that, if $\lambda_{j}>0$, the observed DMU $j$ is efficient. If required, such solutions may be found by the computational method of Podinovski (2007b). The above issue does not affect the radial
} 
resulting conical combination $\tilde{Y}$. The components of this vector are shown in Table 6 . Under the assumption of CRS, it is technologically possible to produce vector $\tilde{Y}$ from the corresponding combination of the input vectors of Farms 28 and 33 (not shown). Taking into account the sign of input inequalities of model (4), we conclude that vector $\tilde{Y}$ can be produced from the input vector of Farm 19.

Observe that, in formula (6), vector $\tilde{Y}$ is subsequently modified by 7 production trade-offs, all of which are assumed to represent technologically possible changes. For example, the trade-off $\bar{Q}_{6}$ allows us to replace 6 tonnes of lucerne by 1 tonne of wheat, and is used in proportion $\pi_{t}=1.54$. Therefore, the output vector $\tilde{Y}+1.54 \bar{Q}_{6}$ is technologically possible, given the input vector of Farm 19.

The next trade-off in formula (6) is $\bar{Q}_{7}$. It states that it is technologically possible to produce at least 1 tonne of wheat instead of 20 tonnes of fodder maize, at any farm in the technology. Using this trade-off in proportion $\pi_{t}=1.01$ as in formula (6), we conclude that the output vector $\tilde{Y}+1.54 \bar{Q}_{6}+1.01 \bar{Q}_{7}$ is technologically possible, given the inputs of Farm 19.

Continuing the above logic, we conclude that the entire vector $\hat{Y}_{19}$ in formula (6) can be produced from the inputs of Farm 19. Table 6 shows the total trade-off $\tilde{Q}=\sum_{t=1}^{K} \pi_{t} Q_{t}$ which is the sum of all 7 trade-off terms in formula (6) taken in the specified proportions $\pi_{t}$. Using this notation, we restate (6) as

$$
\hat{Y}_{19}=\tilde{Y}+\tilde{Q} \text {. }
$$

The sign of output inequalities of model (4) means that the radial target $\theta^{*} Y_{19}=Y_{19}^{*}$ on the right-hand side of program (4) is dominated by the vector $\hat{Y}_{19}$ on its left-hand side. By the free disposability of output assumed in the CRS technology, the vector $Y_{19}^{*}$ can be produced

target $\hat{Y}_{19}$ which is always unique, and the suggested explanation as to why such a target is technologically possible. 
by Farm 19. This demonstrates that the radial target $Y_{19}^{*}$ of Farm 19 as shown in Table 6 is a technologically realistic and producible output vector for this farm. ${ }^{12}$

It is interesting to note that neither Farm 28 nor Farm 33 produces sunflower, which is produced by the inefficient Farm 19. In the conventional CRS model, no conical combination of Farms 28 and 33 could outperform Farm 19. However, by using trade-off $\underline{Q}_{13}$ in proportion $\pi_{t}=2.99$, formula $(6)$ introduces $2.99 \times 0.4 \approx 1.2$ tonnes of sunflower into the product mix of the vector $\hat{Y}_{19}$. This is achieved at the expense of simultaneous reduction of the amount of wheat by 2.99 tonnes. This example shows that farms producing unique crops may be outperformed by farms that do not produce them. The production frontier in this case is induced by the appropriate trade-off that exchanges the commonly produced wheat to the specified amount of the unique crop.

It is worth noting that our treatment of Farms 28 and 33 as the reference set for the inefficient Farm 19 is consistent with a similar interpretation in the conventional VRS and CRS models. An individual DMU from the reference set may, by chance, coincide with the target DMU. In most cases, however, as in our analysis above, the reference DMUs require further modification according to stated production assumptions, to arrive at the target DMU. In the VRS and CRS models this involves taking convex and, respectively, conical combinations of the reference DMUs. In models with production trade-offs permissible modifications also include the use of specified trade-offs.

\section{Conclusion}

Applications of DEA to the analysis of efficiency of DMUs with different specialisations often involve a methodological challenge. In order to construct a meaningful model of technology, all important production inputs and outputs need to be specified. However, because of the specialisation of units, the list of all outputs may be too large, while each output is produced by only a small number of DMUs. In such cases, the application of conventional VRS and CRS models often leads to insufficient efficiency discrimination.

In this paper we propose a way to overcome the above problem based on the incorporation of production trade-offs between different outputs in DEA models. The main

\footnotetext{
${ }^{12}$ Vector $\hat{Y}_{19}$ dominates vector $Y_{19}^{*}$ and generally (as in the standard CRS and VRS models) may not be equal to the latter. In our case, using the data in Table 6 and formula (7), we observe that $\hat{Y}_{19}=Y_{19}^{*}$.
} 
idea of this methodology is to relate the specialised outputs to the main output using production trade-offs. As discussed, this approach has a number of advantages over the other methods, in particular, the conventional aggregation of outputs using their prices. While the latter approach transforms the measurement of technical efficiency to allocative efficiency, the trade-off approach keeps all output dimensions in disaggregated form and allows for the measurement of the technical efficiency of units in the full-dimensional technology.

We illustrate this methodology using an application of DEA to the analysis of efficiency of agricultural farms in eight different regions of Turkey. Each farm produces wheat as the main crop and a number of other crops that only a few other farms produce. We use experts in agricultural production to assess the trade-offs between wheat and the other crops. For example, we ask the experts: how much of barley could be produced with the same resources as needed for the production of one tonne of wheat? The answer is obtained in the form of a range that reflects the uncertainty of experts in their judgement and differences between the farms in the region. The lower and upper bounds of the range allow us to state two trade-offs that link the production of barley to wheat. We do the same with the other crops. Eventually, the specialisation of farms is overcome by relating their specialised outputs to the production of the main crop - wheat.

Computations show that the use of conventional VRS and CRS models in our application results in poor efficiency discrimination due to a large number of outputs in each regional sample. The use of production trade-offs improves the results dramatically, in most regions in our study.

The proposed methodology can be used in other applications of DEA in which DMUs have specialised production profiles. As pointed in the Introduction, examples can be found in different areas, from healthcare to banking.

\section{Acknowledgements}

This research was partly supported by the European Commission through the Seventh Framework Programme (FP7) project 265616, titled "Integrating Econometric and Mathematical Programming Models into an Amendable Policy and Market Analysis Tool using FADN Database" (FADNTOOL). The authors are thankful to Enver Aksoy from Turkish Ministry of Agriculture, Serdar Baltaci from Milas Chamber of Agriculture, and Professor Cemal Polat from Faculty of Agriculture at Namik Kemal University in Turkey, for their collaboration in obtaining the data for this research. 


\section{Appendix}

Below we formulate DEA models for the example considered in Section 3.1. To be specific, consider the assessment of output radial efficiency of farm $B$.

The output-oriented CRS envelopment model (4) with production trade-offs (1) and (2) takes on the following form:

$\max \theta$

subject to

$0 \lambda_{A}+2 \lambda_{B}+4 \lambda_{C}+5 \lambda_{D}-1 \pi_{1}+1 \pi_{2} \geq 2 \theta$

$4.5 \lambda_{A}+4 \lambda_{B}+3 \lambda_{C}+0 \lambda_{D}+0.75 \pi_{1}-1 \pi_{2} \geq 4 \theta$

$1 \lambda_{A}+1 \lambda_{B}+1 \lambda_{C}+1 \lambda_{D}+0 \pi_{1}+0 \pi_{2} \leq 1$

$\lambda_{A}, \lambda_{B}, \lambda_{C}, \lambda_{D}, \pi_{1}, \pi_{2} \geq 0, \theta$ sign free

The first two constraints of model (A.1) correspond to the production of two outputs wheat and barley. The third constraint corresponds to the input - land. Note that both variables $\pi_{1}$ and $\pi_{2}$ are multiplied by zero in the last constraint: this is because $P_{1}$ and $P_{2}$ in trade-offs (1) and (2) are zero vectors.

The CRS multiplier model dual to (A.1) can be stated as follows:

$\min v_{1}$

subject to

$2 u_{1}+4 u_{2}=1$

$1 v_{1}-0 u_{1}-4.5 u_{2} \geq 0$

$1 v_{1}-2 u_{1}-4 u_{2} \geq 0$

$1 v_{1}-4 u_{1}-3 u_{2} \geq 0$

$1 v_{1}-5 u_{1}-0 u_{2} \geq 0$

$-u_{1}+0.75 u_{2} \leq 0$

$u_{1}-u_{2} \leq 0$

$u_{1}, u_{2}, v_{1} \geq 0$

As noted in Section 3.2, the two trade-offs incorporated in model (A.1) are transformed to two weight restrictions in model (A.2). 
The VRS variant of model (A.1) is obtained by the incorporation of the normalising equality $\lambda_{A}+\lambda_{B}+\lambda_{C}+\lambda_{D}=1$. Note that this equality does not include variables $\pi_{1}$ and $\pi_{2}$. The dual multiplier model (A.2) is modified accordingly. 


\section{References}

Acquaye, A.K.A., Alston, J.M., Pardey P.G., 2002. A disaggregated perspective on post-war productivity growth in U.S. agriculture: Isn't that spatial? In: Ball, V.E., Norton, G.W. (Eds.), Agricultural Productivity: Measurement and Sources of Growth. Kluwer Academic Publishers, Boston.

Adler, N., Yazhemsky, E., 2010. Improving discrimination in data envelopment analysis: PCA-DEA or variable reduction. European Journal of Operational Research 202, 273 284.

Alene, A.D., Manyong, V.M., Gockowski, J., 2006. The production efficiency of intercropping annual and perennial crops in southern Ethiopia: A comparison of distance functions and production frontiers. Agricultural Systems 91, $51-70$.

Allen, R., Athanassopoulos, A., Dyson, R.G., Thanassoulis, E., 1997. Weights restrictions and value judgements in data envelopment analysis. Annals of Operations Research 73, $13-34$.

Andersen, P., Petersen, N.C., 1993. A procedure for ranking efficient units in data envelopment analysis. Management Science 39, 1261 - 1264.

Amores, A.F., Contreras, I., 2009. New approach for the assignment of new European agricultural subsidies using scores from data envelopment analysis: Application to olive-growing farms in Andalusia (Spain). European Journal of Operational Research $193,718-729$.

Angulo-Meza, L., Lins, M.P.E., 2002. Review of methods for increasing discrimination in data envelopment analysis. Annals of Operations Research 116, 225 - 242.

Atici, K.B., Podinovski, V.V., 2012. Mixed partial elasticities in constant returns-to-scale production technologies. European Journal of Operational Research 220, 262 - 269.

Brümmer, B., 2001. Estimating confidence intervals for technical efficiency: the case of private farms in Slovenia. European Review of Agricultural Economics 28, 285 - 306.

Cherchye, L., Van Puyenbroeck, T., 2007. Profit efficiency analysis under limited information with an application to German farm types. Omega 35, 335 - 349.

Cooper, W.W., Seiford, L.M., Tone, K., 2007. Data Envelopment Analysis: A Comprehensive Text with Models, Applications, References and DEA-Solver Software. Springer, New York. 
Davidova, S., Latruffe, L., 2007. Relationships between technical efficiency and financial management for Czech Republic farms. Journal of Agricultural Economics. 58, 269 288.

Doyle, J., Green, R, 1994. Efficiency and cross-efficiency in DEA: derivations, meanings and uses. Journal of the Operational Research Society 45, 567 - 578.

EC, European Commission, 2009. Community Committee for the Farm Accountancy Data Network, 2009. Farm Return Data Definitions. RI/CC 1256, rev. 6, Brussels.

EC, European Commission, 2010. Farm Accounting Data Network: An A to Z of Methodology. <http://ec.europa.eu/agriculture/rica//pdf/site_en.pdf > (Accessed in April 2013).

Färe, R., Grabowski, R., Grasskopf, S., 1985. Technical efficiency of Philippine agriculture. Applied Economics 17, 205 - 214.

Färe, R., Grabowski, R., Grasskopf, S., Kraft, S., 1997. Efficiency of a fixed but allocatable input: A non-parametric approach. Economics Letters 56, 187 - 193.

Galanopoulos, K., Aggelopoulos, S., Kamenidou, I., Mattas, K., 2006. Assessing the effects of managerial and production practices on the efficiency of commercial pig farming. Agricultural Systems 88, 125 - 141.

Golany, B., Storbeck, J.E., 1999. A data envelopment analysis of the operational efficiency of bank branches. Interfaces 29, $14-26$.

Iráizoz, B., Rapún, M., Zabaleta, I., 2003. Assessing the technical efficiency of horticultural production in Navarra, Spain. Agricultural Systems 78, 387 - 403.

Latruffe, L., Balcombe, K., Davidova, S., Zawalinska, K., 2004. Determinants of technical efficiency of crop and livestock farms in Poland. Applied Economics 36, 1255 - 1263.

Liu, J.S., Lu, L.Y.Y., Lu, W.-M., Lin, B.J.Y., 2013. A survey of DEA applications. Omega $41,893-902$.

Luik, H., Seilenthal, J., Värnik, R., 2009. Measuring the input-orientated technical efficiency of Estonian grain farms in 2005-2007. Food Economics - Acta Agriculturae Scandinavica, Section C, 6, $204-210$.

Olesen, O.B., Petersen, N.C., 2002. The use of data envelopment analysis with probabilistic assurance regions for measuring hospital efficiency. Journal of Productivity Analysis 17, 83-109.

Oude Lansink, A., Pietola, K., Bäckman, S., 2002. Efficiency and productivity of conventional and organic farms in Finland 1994-1997. European Review of Agricultural Economics 29, $51-65$. 
Ozcan, Y.A., 2008. Health Care Benchmarking and Performance Evaluation. An Assessment Using Data Envelopment Analysis (DEA). Springer, New York, NY.

Paul, C.M., Nehring, R., Banker, D., Somwaru, A., 2004. Scale economies and efficiency in U.S. Agriculture: Are traditional farms history? Journal of Productivity Analysis 22, $185-205$.

Podinovski, V.V., 2004. Production trade-offs and weight restrictions in data envelopment analysis. Journal of the Operational Research Society 55, 1311 - 1322.

Podinovski, V.V., 2007a. Improving data envelopment analysis by the use of production trade-offs. Journal of the Operational Research Society 58, 1261 - 1270.

Podinovski, V.V., 2007b. Computation of efficient targets in DEA models with production trade-offs and weight restrictions. European Journal of Operational Research 181, 586 -591 .

Podinovski, V.V., Bouzdine-Chameeva, T., 2013. Weight restrictions and free production in data envelopment analysis. Operations Research 61, 426 - 437.

Podinovski, V.V., Førsund, F.R., 2010. Differential characteristics of efficient frontiers in data envelopment analysis. Operations Research 58, 1743 - 1754.

Podinovski V.V., Thanassoulis, E., 2007. Improving discrimination in data envelopment analysis: some practical suggestions. Journal of Productivity Analysis 28, $117-126$.

Ramón , N., Ruiz, J.L., Sirvent, I., 2011. Reducing differences between profiles of weights: A “peer-restricted"' cross-efficiency evaluation. Omega 39, $634-641$.

Ray, S.C., Bhadra, D., 1993. Nonparametric test of cost minimizing behavior: A study of Indian farms. American Journal of Agricultural Economics 75, 990 - 999.

Reinhard, S., Lovell, C.A.K., Thijssen, G.J., 2000. Environmental efficiency with multiple environmentally detrimental variables; estimated with SFA and DEA. European Journal of Operational Research 121, 287 - 303.

Saçli, Y., 2009. Agricultural Statistics in Turkey: Evolution, Problems and Solutions. Turkish Ministry of Development, Ankara, Turkey.

Skevas, T., Oude Lansink, A., Stefanou, S.E., 2012. Measuring technical efficiency in the presence of pesticide spillovers and production uncertainty: The case of Dutch arable farms. European Journal of Operational Research 223, 550 - 559.

Speelman., S., D’Haese, M., Buysse, J., D’Haese, L., 2008. A measure for the efficiency of water use and its determinants, a case study of small-scale irrigation schemes in NorthWest Province, South Africa. Agricultural Systems 98, 31 - 39. 
Thanassoulis, E., 1999. Data envelopment analysis and its use in banking. Interfaces 29, 1 13.

Thanassoulis, E., 2001. Introduction to the Theory and Application of Data Envelopment Analysis. Kluwer Academic Publishers, Dordrecht.

Thanassoulis, E., Portela, M.C.S., Despić, O., 2008. Data envelopment analysis: The mathematical programming approach to efficiency analysis. In: Fried, H.O., Lovell, C.A.K., Schmidt, S.S. (Eds.), The Measurement of Productive Efficiency and Productivity Growth. Oxford University Press, New York, pp. $251-420$.

Thomas, A.C., Tauer, L., 1994. Linear input aggregation bias in nonparametric technical efficiency measurement. Canadian Journal of Agricultural Economics 42, 77 - 86. 